\title{
A Tool to Improve Accuracy of Parental Measurements of Preschool Child Height
}

\author{
Meredith Yorkin, ${ }^{1}$ Kim Spaccarotella, ${ }^{1,2}$ Jennifer Martin-Biggers, ${ }^{1}$ Carolina Lozada, ${ }^{1}$ \\ Nobuko Hongu, ${ }^{3}$ Virginia Quick, ${ }^{4}$ and Carol Byrd-Bredbenner ${ }^{1}$ \\ ${ }^{1}$ Department of Nutritional Sciences, Rutgers, The State University of New Jersey, New Brunswick, NJ 08901, USA \\ ${ }^{2}$ Department of Biological Sciences, Kean University, Union, NJ 07083, USA \\ ${ }^{3}$ Department of Nutritional Sciences, University of Arizona, Tucson, AZ 85721, USA \\ ${ }^{4}$ Department of Health Sciences, James Madison University, Harrisonburg, VA 22807, USA
}

Correspondence should be addressed to Jennifer Martin-Biggers; jmartin@njaes.rutgers.edu

Received 3 May 2015; Revised 15 July 2015; Accepted 16 July 2015

Academic Editor: Livio Pagano

Copyright (C) 2015 Meredith Yorkin et al. This is an open access article distributed under the Creative Commons Attribution License, which permits unrestricted use, distribution, and reproduction in any medium, provided the original work is properly cited.

Background. Parent-reported measurement of child height is common in public health research but may be inaccurate, especially for preschoolers. A standardized protocol and tools to improve measurement accuracy are needed. The purpose of this study was to develop and test materials to improve parents' accuracy when measuring their preschooler's height. Methods. In Phase A, 24 parents were observed measuring child height using written instructions and an easy-to-read tape measure; after each of 3 testing rounds, instructions were refined based on observed errors and parent versus researcher measurements. In Phase B, a video replaced written instructions and was refined over 4 rounds with 37 parents. Results. The height kit with written instructions, tape measure, plumb line, and explanatory video helped parents accurately measure child height. Compared to written instructions alone, parents rated the video as having significantly greater clarity and likelihood of improving measurements. Although no significant differences in accuracy were found between paper and video instructions, observations indicated written instructions were more difficult for parents with less education to use with fidelity. Conclusions. The kit may improve parent measurement of preschooler height, thereby improving accuracy of body mass index calculations, tracking of obesity prevalence, and obesity prevention and treatment.

\section{Introduction}

Body mass index (BMI) is calculated using body weight and height measurements. Parent-reported measurement of child's height and weight is often used in public health research because this data collection method is efficient and cost-effective $[1,2]$. However, parent reports of child height may not be accurate, especially for young children $[3,4]$. In a study of parent-reported weights and heights of themselves and their obese children, a fifth of children's heights were overestimated by more than $2.5 \mathrm{~cm}(\sim 1$ in) [5]. Similarly, a more recent study of 837 children reported that $16.5 \%$ of parents underestimated and $25 \%$ overestimated child height by at least $2.5 \mathrm{~cm}(\sim 1 \mathrm{in})$ [6]. Another study found that mothers' overestimation of both child weight and height resulted in an overestimation of the percentage of overweight children by more than $3 \%$ in a sample of 1,549 4-year-olds [7].

Inaccuracies in parent reports of child height may be due to difficulty measuring young children due to their small size, children's difficulty in standing still during measurement procedures, parent use of incorrect measuring techniques, parent reliance on recall of height data from a doctor's visit, and parent reporting bias [4]. In addition, parents may incorrectly report their own height, which causes errors in calculations of their children's target height. A study of 241 families found that only about half of the parents reported their height within $\pm 2 \mathrm{~cm}$ of their measured height, and only $70 \%$ of the midparental target heights calculated with the parentreported data were accurate within $\pm 2 \mathrm{~cm}$ of the target heights (i.e., the predicted height of the child based on height of 
both parents) calculated from measured data [8]. Clinicians often used target height calculations when assessing children's growth trajectories, making height an important component of pediatric anthropometric assessment [8].

Although these inaccuracies make it difficult to have confidence in parent-reported child height data when tracking individual children, parental reports of child height and weight may be acceptably accurate for estimating obesity prevalence in populations. A comparison of measured obesity prevalence in 1,497 school children with overweight prevalence estimated from parent-reported height and weight data found that both were similar, despite the tendency of parents to overestimate overweight by about $17 \%$ in boys and $10 \%$ in girls [9].

The obesity epidemic has spawned increased parent interest in child growth as well as obesity prevention intervention studies using BMI as an outcome measure [10-12]. Due to logistical issues associated with geographic distances between intervention participants and researchers, increased use of Internet-based interventions and data collection methods, funding limitations, and participant convenience, a standardized child height measurement protocol and tools designed for parents that could improve measurement accuracy are needed [13]. Improved height measurement tools would also increase the confidence in obesity prevention intervention results. Thus, the purpose of this study was to develop and pilot-test materials to help parents improve the accuracy with which they measured their preschool child's height.

\section{Materials and Methods}

This study was approved by the Institutional Review Boards at Rutgers University and the University of Arizona. All participants gave informed consent.

2.1. Sample. Parents of 2- to 5-year-old children were recruited via flyers posted at community sites and emails sent through workplace listservs at Rutgers University and the University of Arizona. Recruitment notices invited parents to review materials, participate in an interview, and implement instructions for measuring their own and their preschool child's height. Each participating parent received \$25 compensation, and children received a sheet of stickers to compensate them for their time. No parent-child dyad participated in more than one round of testing.

\subsection{Development of Height Measurement Kit. An iterative} process was used to develop materials to improve the accuracy with which parents measured and reported child height. The height protocol for parents was created in two phases. Participants in both phases completed a brief survey to gather demographic data (e.g., age, highest education level achieved).

2.3. Phase A. Brief, written instructions for measuring height were developed by nutrition researchers with extensive experience conducting anthropometric measurements. Drafts were iteratively reviewed, tested, and refined by a panel of experts in anthropometric measurements $(n=3)$ to ensure accuracy and nonexperts $(n=8)$ to ensure comprehension by inexperienced audiences. The instructions described how to hang the tape measure and how to measure height accurately (Figure 1). Research staff then prepared height kits that contained a measuring tape, plumb line (called a weighted string in the instructions, made by tying a $1.27 \mathrm{~cm}(1 / 2 \mathrm{in})$ flat washer to a length of mason's line), removable tape for hanging the tape measure and plumb line, and written instructions.

A vast array of commercially available tape measures were reviewed to determine which would have the greatest likelihood of yielding the most accurate measurements. Most were narrow (less than $2.5 \mathrm{~cm}$ or 1 in wide) and had measurements (tick marks) in increments of $1 / 16$ th in $(0.16 \mathrm{~cm})$. These were judged by the research staff as cost-effective but difficult to read accurately when hanging on a wall and had a precision level beyond that needed to calculate body mass index (BMI) percentile. Thus, a $7.6 \mathrm{~cm}$ wide (3 in) tape measure similar to those used by visually impaired individuals with increments marked at $1 / 4$ th in $(0.63 \mathrm{~cm})$ was created using a poster printer; these dimensions and precision level were selected to make it easier for parents to read and differentiate between fractions. In addition, this tape measure labeled foot increments and restarted numbering of inches at each foot marker (i.e., the measurements were $1,2,3,4,5,6,7,8,9,10$, and 11 inches, 1 foot, $1,2,3,4,5,6,7,8,9,10$, and 11 inches, 2 feet, and so on) so that parents could report height in feet and inches (e.g., 3 feet, 2 inches), which is customary in the United States.

The height kits were subjected to three rounds of testing in Phase A. In all rounds, researchers explained to parents that they planned to mail the height kits to parents in an upcoming Internet-based study and wanted to be certain the instructions for measuring height were clear. The researchers further explained that the purpose of parents' participation was to help researchers improve the kits; thus parents should act as though they were in their own homes and the researchers were not there to answer questions. Each parent then was asked to read and follow the instructions and report the height measurements they obtained for their preschool child who accompanied them to the testing site. Then, with the parent watching, the researcher removed the measuring tape and plumb line, rehung the tape measure and plumb line in the same location used by the parent (if appropriate) or another location (if necessary to avoid obstacles that would result in erroneous measurements such as baseboards and carpet), and measured the child in duplicate. Finally, parents were asked for suggestions for improving the instructions and to use a 5-point scale, with 5 being the best score, to rate the instructions' clarity, readability, and likelihood that they would improve the way they measure their children's height.

Two trained researchers observed each parent as they operationalized the instructions to identify errors made in height measurement procedures. These errors were analyzed and used to refine the height kit prior to the next round of testing. 


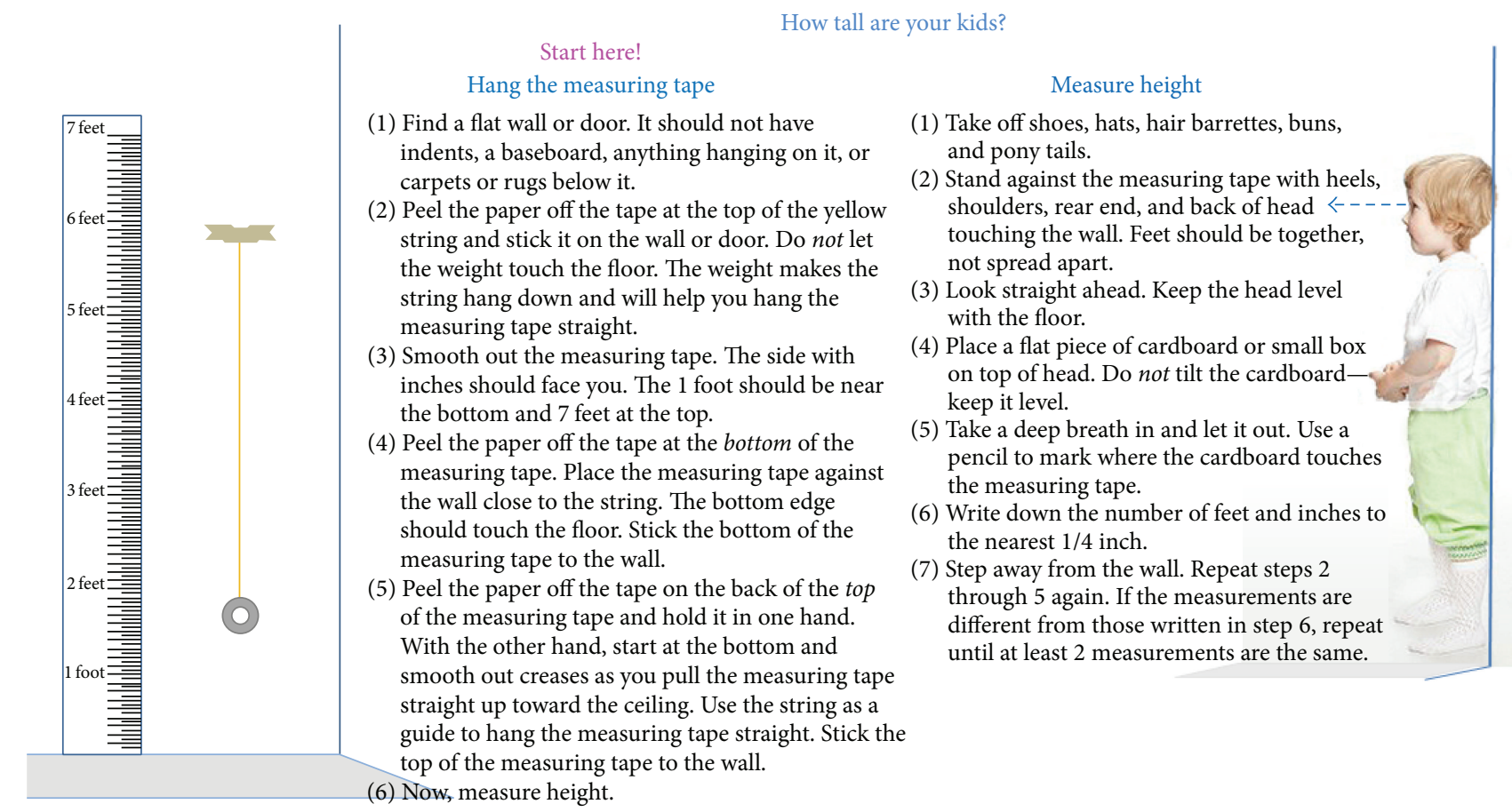

FIGURE 1: Height measurement protocol.

2.4. Phase B. The need for Phase B became apparent with the findings from Phase A (see Results and Discussion). In Phase $B$, the research team created a brief (8-minute) narrated video (http://healthyhomestyles.com/height/). The video was developed using Phase A's written instructions and was reviewed by a panel of experts in anthropometric measurements $(n=3)$ and nonexperts $(n=5)$. The video had components that described and depicted how to select a suitable location for hanging the tape measure, purpose and importance of the weighted string, how to ensure the tape measure was hung straight on the wall, how to prepare the child for being measured (e.g., remove shoes, hats, and hair decorations), positioning the child for measurement (having child's head, shoulders, buttocks, and heals touch the wall while child looks straight ahead), importance of child inhaling and exhaling before measurement, recording height to the nearest $1 / 4$ in $(0.63 \mathrm{~cm})$, and importance of taking measurements twice.

The video and height kits were iteratively tested and refined in a series of four rounds of testing in Phase B. The video, played at research sites on a standalone laptop computer, was accompanied by a height kit developed and refined in Phase A. The procedures mirrored those used in Phase A. In addition, the survey completed at the start of the data collection protocol asked parents to report their child's height and indicate how sure they were of the reported height using a 5-point scale. Parents also were interviewed by trained researchers at the end of the session to identify questions that arose as they watched each component of the video and suggestions for improving the understandability of the video. Finally, parents used a 5 -point scale, with 5 being the best score, to rate the clarity of the instructions in the video, how well the video held their attention, and likelihood that the video would improve their measurement of child height.

2.5. Statistical Analysis. For each participant, the difference between mean height as measured by the parent and the researcher "gold standard" was calculated and compared. $t$ tests were used to compare height measurements made by research staff with those made by parents. Differences were considered significant at $P<0.05$. Values are reported as means and standard deviations (SD) unless otherwise noted.

\section{Results and Discussion}

3.1. Results: Phase A. Table 1 describes participating parents' mean ages, education level, and number of children living in their households. Six parent-child dyads recruited from a university-based preschool participated in Round 1 of testing. As shown in Table 2, parents rated the instructions as being clear, easy to read, and likely to improve their measurements of child height. A comparison of height measurements taken by parents with those of researchers revealed close agreement in child heights. Instructions were refined based on parent feedback and consistent errors noted by researchers. The key error noted during this round was that parents did not understand the use of the plumb line and did not always choose a flat wall (many hung the tape measure over a baseboard). Additionally, many parents had difficulty differentiating between the fractions and accurately stating child height.

In Round 2, 13 parent-child dyads recruited from a university-based preschool participated. As in Round 1, parents had a mean age of about 38 years and all had at least some postsecondary education. Similarly, parents rated the clarity 
TABLE 1: Characteristics of participants and accuracy of child height measurements.

\begin{tabular}{|c|c|c|c|c|c|}
\hline \multirow{2}{*}{$\begin{array}{l}\text { Phase } \\
\text { Round }\end{array}$} & \multirow{2}{*}{$\begin{array}{l}\text { Parent's age } \\
\text { in years } \\
\text { Mean } \pm \text { SD }\end{array}$} & \multicolumn{2}{|c|}{ Education } & \multirow{2}{*}{$\begin{array}{c}\text { Number of } \\
\text { children }<18 \text { in } \\
\text { household } \\
\text { Mean } \pm \text { SD }\end{array}$} & \multirow{2}{*}{$\begin{array}{l}\text { Absolute mean difference in } \\
\text { inches }(\mathrm{cm}) \text { between parent and } \\
\text { researcher measured child } \\
\text { heights } \\
\text { Mean } \pm \mathrm{SD}\end{array}$} \\
\hline & & $\begin{array}{c}\text { Percent with } \\
\text { secondary } \\
\text { education or less }\end{array}$ & $\begin{array}{c}\text { Percent with } \\
\text { postsecondary } \\
\text { education }\end{array}$ & & \\
\hline \multicolumn{6}{|l|}{ Phase A } \\
\hline Round $1(n=6)$ & $38.80 \pm 6.15$ & $0 \%$ & $100 \%$ & $2.16 \pm 0.41$ & $\begin{array}{c}0.23 \pm 0.23 \\
(0.58 \pm 0.58)\end{array}$ \\
\hline Round $2(n=13)$ & $38.38 \pm 4.23$ & $0 \%$ & $100 \%$ & $2.00 \pm 0.82$ & $\begin{array}{c}0.60 \pm 0.65 \\
(1.52 \pm 1.65)^{\mathrm{a}}\end{array}$ \\
\hline Round $3(n=5)$ & $\mathrm{b}$ & $100 \%$ & $0 \%$ & b & $\begin{array}{c}1.38 \pm 2.12 \\
(3.51 \pm 5.38)\end{array}$ \\
\hline \multicolumn{6}{|l|}{ Phase B } \\
\hline Round $1(n=9)$ & $31.78 \pm 8.91$ & $44 \%$ & $66 \%$ & $2.44 \pm 1.33$ & $\begin{array}{c}1.66 \pm 1.97 \\
(4.22 \pm 5.00)\end{array}$ \\
\hline Round $2(n=13)$ & $35.67 \pm 12.48$ & $54 \%$ & $46 \%$ & $1.42 \pm 0.51$ & $\begin{array}{c}0.71 \pm 0.99 \\
(1.80 \pm 2.51)^{\mathrm{c}}\end{array}$ \\
\hline Round $3(n=5)$ & $28.60 \pm 6.80$ & $40 \%$ & $60 \%$ & $3.00 \pm 1.58$ & $\begin{array}{c}1.55 \pm 1.04 \\
(3.94 \pm 2.64)\end{array}$ \\
\hline Round $4(n=10)$ & $29.11 \pm 6.17$ & $50 \%$ & $50 \%$ & $1.70 \pm 0.82$ & $\begin{array}{c}0.35 \pm 0.40 \\
(0.89 \pm 1.02)\end{array}$ \\
\hline
\end{tabular}

${ }^{\mathrm{a}} n=11$.

${ }^{\mathrm{b}}$ Data unavailable.

${ }^{c} n=12$.

TABle 2: Parent rating of height kit.

\begin{tabular}{|c|c|c|c|c|}
\hline $\begin{array}{l}\text { Phase } \\
\text { Round }\end{array}$ & $\begin{array}{c}\text { Clarity of } \\
\text { instructions }\end{array}$ & Reading ease ${ }^{* a}$ & $\begin{array}{l}\text { Extent video held } \\
\text { viewer's attention*b }\end{array}$ & $\begin{array}{l}\text { Likelihood of } \\
\text { improving } \\
\text { measurement of } \\
\text { child's height }^{*}\end{array}$ \\
\hline \multicolumn{5}{|l|}{ Phase A } \\
\hline Round $1(n=6)$ & $4.50 \pm 0.55$ & $4.67 \pm 0.52$ & - & $4.00 \pm 1.55$ \\
\hline Round $2(n=13)^{c}$ & $4.50 \pm 0.90$ & $4.50 \pm 0.90$ & - & $3.92 \pm 1.08$ \\
\hline Round $3(n=5)$ & $4.65 \pm 0.49$ & $5.00 \pm 0.00$ & - & $4.60 \pm 0.89$ \\
\hline All Phase A rounds combined & $4.53 \pm 0.72$ & $4.65 \pm 0.71$ & & $4.09 \pm 1.16$ \\
\hline \multicolumn{5}{|l|}{ Phase B } \\
\hline Round $1(n=9)$ & $4.78 \pm 0.44$ & - & $3.94 \pm 1.07$ & $5.00 \pm 0.00$ \\
\hline Round $2(n=13)$ & $4.92 \pm 0.28$ & - & $4.54 \pm 0.28$ & $5.00 \pm 0.00$ \\
\hline Round $3(n=5)$ & $4.80 \pm 0.45$ & - & $4.00 \pm 1.00$ & $5.00 \pm 0.00$ \\
\hline Round $4(n=10)$ & $4.90 \pm 0.32$ & - & $4.80 \pm 0.42$ & $4.95 \pm 0.16$ \\
\hline All Phase B rounds combined & $4.86 \pm 0.35^{\mathrm{d}}$ & & $4.39 \pm 0.86$ & $4.98 \pm 0.08^{\mathrm{e}}$ \\
\hline
\end{tabular}

${ }^{*}$ Item rated using a 1-to-5-point scale with 5 being the best score.

${ }^{a}$ Item included in Phase A only.

${ }^{\mathrm{b}}$ Item included in Phase B only.

${ }^{c}$ One missing response.

${ }^{\mathrm{d}}$ Significantly higher than Phase A $(P=0.002)$.

${ }^{\mathrm{e}}$ Significantly higher than Phase A $(P=0.0004)$. 
of instructions and reading ease highly but were less positive that the height kit would improve the accuracy of the height measurements they made of their children. A comparison of researcher and parent child height measurements revealed close agreement. Minor refinements were made to the instructions based on parent comments and researcher observations.

In Round 3, 5 parent-child dyads were recruited from a community center. None of these parents had any postsecondary education. Although parents felt the instructions were clear, easy to read, and likely to improve their measurements of child height, researcher observations revealed that written instructions were difficult for parents with less education to use with fidelity. Mean researcher and parent child height measurements differed by 1.38 in $(3.51 \mathrm{~cm})$.

3.2. Results: Phase B. In Rounds 1 to 4 of Phase B, 9, 13, 5, and 10 parent-child dyads participated, respectively. Of these, about half had no postsecondary education. Common errors were not verifying tape measure straightness, hanging the tape measure over a baseboard instead of finding a flat wall, adjusting straightness of the tape measure by moving the bottom instead of the top which resulted in the tape not remaining flush with the floor, not removing child shoes, not having child breathe in/out before taking measurements, and misreading fractions. Several parents also suggested that the video should end with a summary of the steps and be accompanied with written instructions (i.e., "some kind of cue card at the end to remind you of all of the steps") and that a reminder to start hanging the tape from the bottom should be added ("more emphasis on remembering to tape the bottom of the measuring tape first"). The video was refined iteratively after each round to address consistent parent errors and suggestions by emphasizing the importance of procedures, refining text and images appearing in the video, repeating the purpose of procedures (e.g., hanging the plumb line), and adding a summary of procedures. In addition, to improve accuracy in reading fractions, the measuring tape was modified in each round, respectively, by increasing the font size of the numbers, renumbering so that the foot increments were removed and inches ran consecutively, introducing a yellow background to make the increments and numbers more visible, and adding written instructions to the measuring tape itself. To emphasize that the tape measure must touch the floor, a thick black line was added to the bottom of the tape measure and the bottom and top of the tape measure were labeled. Throughout Phase B, parents highly rated the video's clarity, ability to hold viewer attention, and likelihood of improving their measurements of child height. In the final round, mean differences in child height measurements of researchers and parents were approximately $1 / 3$ in $(0.85 \mathrm{~cm})$. The final components of the height kit are shown in Figure 2.

Interestingly, on the survey completed at the outset of data collection, 18 (49\%) parents reported that they did not know their child's height. Average absolute differences between the values of the parents who reported child height $(n=19)$ and researchers' measurements were $5.37 \pm 4.31 \mathrm{SD}$ in or $13.6 \pm$ $10.9 \mathrm{SD} \mathrm{cm}$ (range 0.70 to 15.3 in or 1.8 to $38.9 \mathrm{~cm}$ ). One

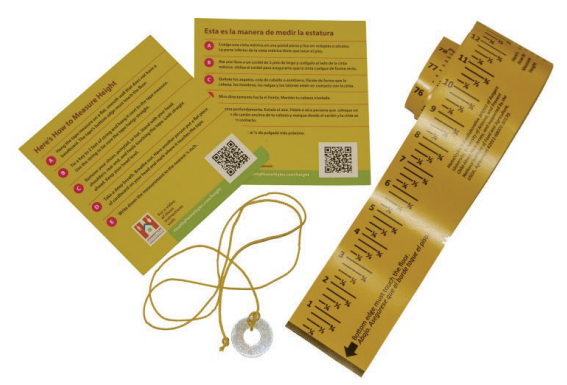

FIGURE 2: Height measurement kit. Video: http://healthyhomestyles .com/height/.

parent in 19 who reported feeling sure about the child height reported underreported the child's height by 1.75 in $(4.45 \mathrm{~cm})$.

Comparisons, using $t$-tests, of instruction clarity ratings for all rounds of Phase A combined with all rounds of Phase $\mathrm{B}$ combined revealed that participants rated the video as having significantly greater clarity $(P=0.0204)$ than written instructions. Similarly, participants rated the video as significantly more likely to improve their measurements of child heights than the written instructions $(P=0.0004)$. Average absolute differences in child height measurements between researchers and parents for all Phase A and Phase $\mathrm{B}$ rounds (i.e., $0.67 \pm 1.32 \mathrm{SD}$ and $0.96 \pm 1.29 \mathrm{SD}$ in or $1.70 \pm 3.34 \mathrm{SD}$ and $2.44 \pm 3.28 \mathrm{SD} \mathrm{cm}$, resp.) did not differ significantly $(P=0.1879)$. Additionally, in Phase $\mathrm{B}$, there were no significant differences between those with high school education levels or less and those with at least some postsecondary education with regard to accuracy of child height measurements compared to those taken by researchers $(1.09 \pm 1.52 \mathrm{SD}$ versus $0.81 \pm 1.00 \mathrm{SD}$ in or $2.77 \pm 3.86 \mathrm{SD}$ versus $2.06 \pm 2.52 \mathrm{SD} \mathrm{cm}, P=0.2533$ ) (sample size was too small to conduct similar comparisons in Phase A).

\section{Discussion}

This study's findings indicate that a height kit composed of written instructions, an easy-to-read tape measure, plumb line, and explanatory video can help parents accurately measure child height. Compared to written instructions alone, parents rated the video as having significantly greater clarity and likelihood of improving their measurements of child height. Although no significant differences in accuracy were found between the written and video instructions, researcher observations indicated that the written instructions alone were more difficult for parents to use with fidelity, especially those with less formal education.

Self-report data are considered to be an accurate representation only when the data collection instrument has undergone formative testing such as reliability and validity analysis [14]. Adults are generally accurate when providing their own height and weight to calculate BMI $[15,16]$ (with the exception of overweight and obese women tending to underreport weights) [17]; however, preschool-aged children 
are rapidly growing and parents frequently misreport both height and weight $[6,7]$. Even if a child recently had a pediatric or well-child visit, substantial amounts of growth can happen in a relatively short time frame, and reporting of this data may not be sufficiently accurate for tracking individual differences in growth [3]. The height kit designed and refined in this study can help researchers cost-effectively gather more accurate parent-reported data on child height. Kits cost less than $\$ 2.00$ US each and can be mailed to study participants.

The small sample sizes in this study indicate that statistical comparison should be interpreted with caution. In addition, parents took measurements while under observation and may not be as careful in taking measurements when at home. However, the study has numerous strengths. An iterative process was used to continually modify and refine the measuring tape and written and video instructions and a mixed methods approach was taken for data collection. Quantitative measures of height measurements taken by parents were compared to the researcher "gold standard" and qualitative data collection from parents was used to identify specific areas of confusion and areas for improving and refining the written instructions and video. Future research should investigate the effectiveness of the height kit in improving parental height measurements of older children and determine its utility in more diverse population groups. Pairing the height measurements taken with the kit and child weight could further clarify usefulness of the height kit and video in reporting BMI.

\section{Conclusions}

Study findings suggest that an instructional video is perceived by parents as clear, easy to use, and likely to improve their measurements of child height. The height measuring kit developed and validated in this study has the potential to improve parent reports of child height, thereby improving the accuracy of BMI calculations, tracking of childhood obesity prevalence, and confidence in obesity prevention and treatment program outcomes based on parent-reported data.

\section{Conflict of Interests}

The authors declare that they have no competing interests.

\section{Authors' Contribution}

The following coauthors contributed to the work: Meredith Yorkin to study design, data collection, paper preparation, and paper review; Kim Spaccarotella to paper preparation, data analysis, and paper review; Jennifer Martin-Biggers to study design, data collection, data analysis, and paper review; Carolina Lozada to study design, data collection, and paper review; Nobuko Hongu to data collection and paper preparation and review; Virginia Quick to data analysis, paper preparation, and paper review; Carol Byrd-Bredbenner to study design and paper preparation and paper review. All authors read and approved the final paper.

\section{Funding}

This work was funded through USDA NIFA \#2011-6800130170.

\section{Acknowledgment}

Meredith Yorkin, Kim Spaccarotella, Jennifer Martin-Biggers, Virginia Quick, and Carol Byrd-Bredbenner received funding from the United States Department of Agriculture, National Institute of Food and Agriculture, Grant no. 201168001-30170.

\section{References}

[1] I. Huybrechts, J. H. Himes, C. Ottevaere et al., "Validity of parent-reported weight and height of preschool children measured at home or estimated without home measurement: a validation study," BMC Pediatrics, vol. 11, article 63, 2011.

[2] N.-L. Yao and M. M. Hillemeier, "Weight status in Chinese children: maternal perceptions and child self-assessments," World Journal of Pediatrics, vol. 8, no. 2, pp. 129-135, 2012.

[3] A. C. Skinner, D. Miles, E. M. Perrin, T. Coyne-Beasley, and C. Ford, "Source of parental reports of child height and weight during phone interviews and influence on obesity prevalence estimates among children aged 3-17 years," Public Health Reports, vol. 128, no. 1, pp. 46-53, 2013.

[4] L. J. Akinbami and C. L. Ogden, "Childhood overweight prevalence in the United States: the impact of parent-reported height and weight," Obesity, vol. 17, no. 8, pp. 1574-1580, 2009.

[5] R. R. Wing, L. H. Epstein, and D. Neff, "Accuracy of parents' reports of height and weight," Journal of Behavioral Assessment, vol. 2, no. 2, pp. 105-110, 1980.

[6] D. P. O’Connor and J. J. Gugenheim, "Comparison of measured and parents' reported height and weight in children and adolescents," Obesity, vol. 19, no. 5, pp. 1040-1046, 2011.

[7] L. Dubois and M. Girad, "Accuracy of maternal reports of preschoolers' weights and heights as estimates of BMI values," International Journal of Epidemiology, vol. 36, no. 1, pp. 132-138, 2007.

[8] I. Braziuniene, T. A. Wilson, and A. H. Lane, "Accuracy of selfreported height measurements in parents and its effect on midparental target height calculation," BMC Endocrine Disorders, vol. 7, article 2, 2007.

[9] A. Banach, T. J. Wade, J. Cairney, J. A. Hay, B. E. Faught, and D. D. O'Leary, "Comparison of anthropometry and parentreported height and weight among nine year olds," Canadian Journal of Public Health, vol. 98, no. 4, pp. 251-253, 2007.

[10] C. Byrd-Bredbenner, J. Worobey, J. Martin-Biggers et al., "HomeStyles: shaping home environments and lifestyle practices to prevent childhood obesity: a randomized controlled trial," Journal of Nutrition Education and Behavior, vol. 44, no. 4, supplement, p. S81, 2012.

[11] J. Emerson, B. Husaini, P. Hull, R. Levine, and V. Oates, "Nashville children eating well (CHEW) for health," Journal of Nutrition Education and Behavior, vol. 44, no. 4, supplement, p. S81, 2012.

[12] O. Seaver and R. Mullis, "Family food and fitness fun pack: a pilot study," Journal of Nutrition Education and Behavior, vol. 44, no. 4, supplement, pp. S27-S28, 2012. 
[13] M. Yorkin, K. Spaccarotella, J. Martin-Biggers, V. Quick, and C. Byrd-Bredbenner, "Accuracy and consistency of weights provided by home bathroom scales," BMC Public Health, vol. 13, article 1194, 2013.

[14] C. Redding, J. Maddock, and J. Rossi, “The sequential approach to measurement of health behavior constructs: issues in selecting and developingmeasures," Californian Journal of Health Promotion, vol. 4, no. 1, pp. 83-101, 2006.

[15] W. Willett, Nutritional Epidemiology, Oxford University Press, New York, NY, USA, 2nd edition, 1998.

[16] M. F. Kuczmarski, R. J. Kuczmarski, and M. Najjar, "Effects of age on validity of self-reported height, weight, and body mass index: findings from the third National Health and Nutrition Examination Survey, 1988-1994," Journal of the American Dietetic Association, vol. 101, no. 1, pp. 28-34, 2001.

[17] M. J. Kretsch, A. K. H. Fong, and M. W. Green, "Behavioral and body size correlates of energy intake underreporting by obese and normal-weight women," Journal of the American Dietetic Association, vol. 99, no. 3, pp. 300-306, 1999. 


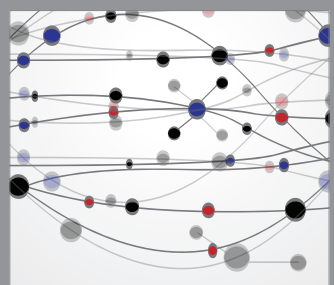

The Scientific World Journal
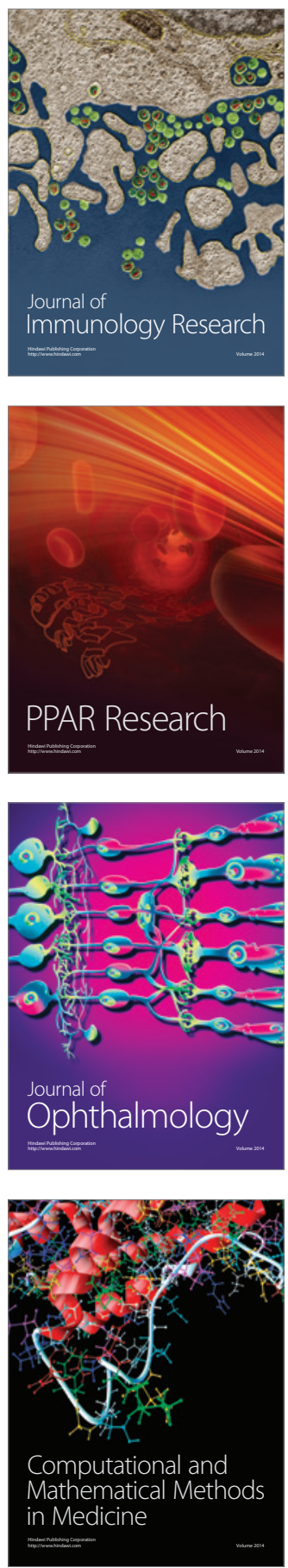

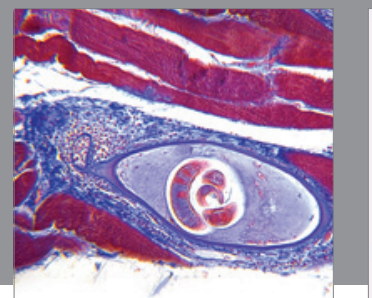

Gastroenterology

Research and Practice
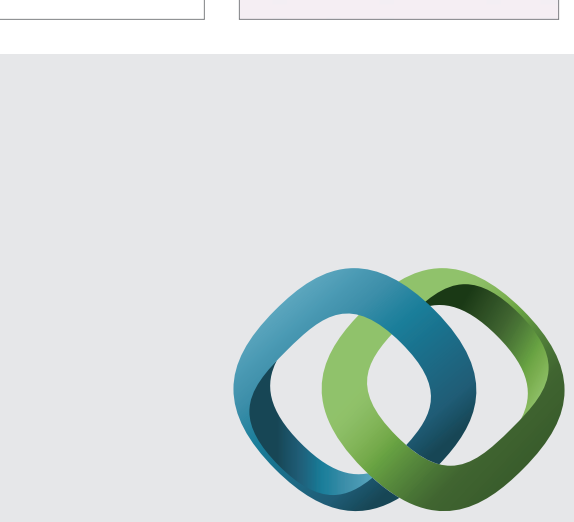

\section{Hindawi}

Submit your manuscripts at

http://www.hindawi.com
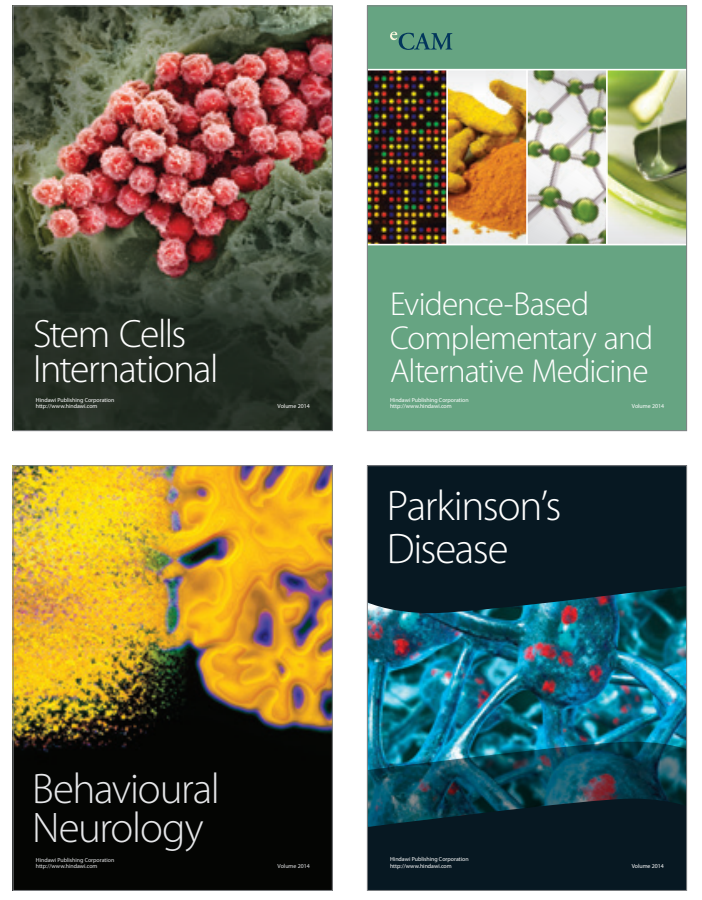
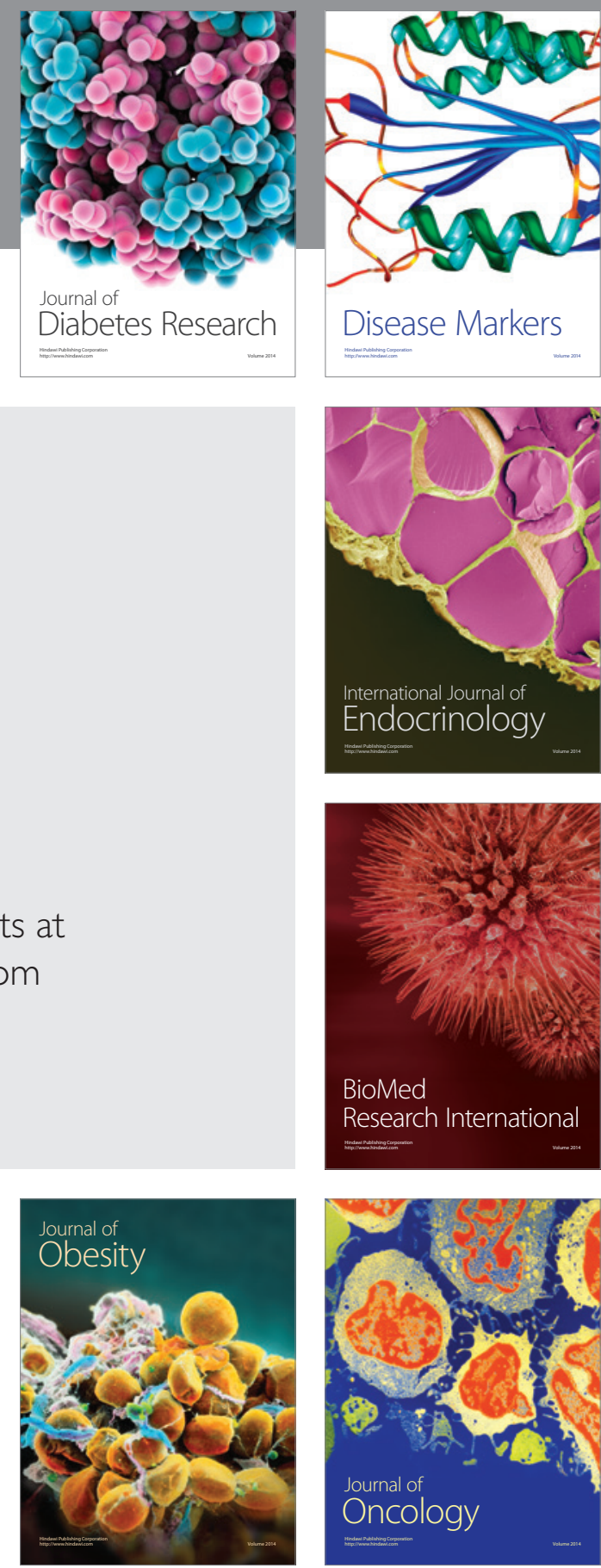

Disease Markers
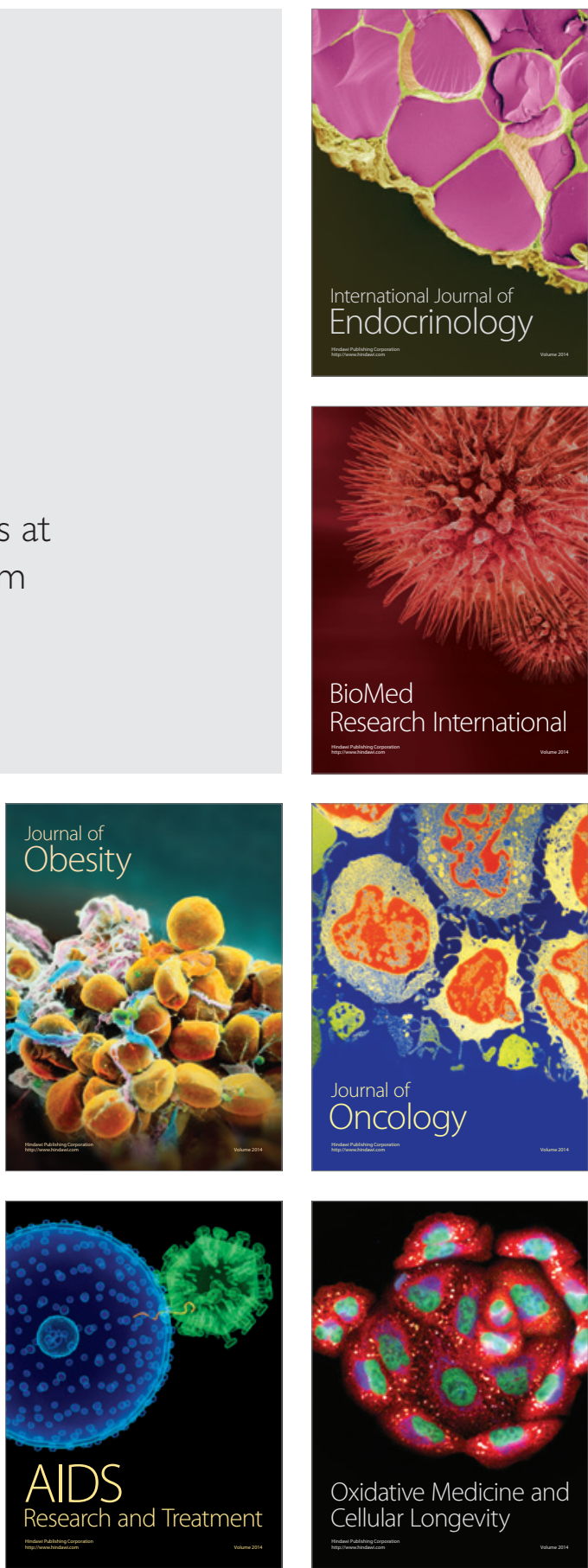